\title{
Statement of Peer review
}

In submitting conference proceedings to KnE publishing, the conference editors confirm the following:

- They adhere to Knowledge E Policy on Publication Ethics and Publication Malpractice Statement

- All submitted conference papers have passed a peer review process conducted by the Chief Academic Editor of the conference

- Please provide a description of your conference peer review process, type (single-blind, double-blind, etc.), and state the total number of peer reviewers:

The type of review process were double-blinded and the total number of peer reviewer is one for one paper with 15 reviewers

- All the editors have taken all mandatory steps to ensure the quality of the manuscripts they publish, and their decision to accept or reject a manuscript for publication has been based only on the quality of the produced work.

Conference full title: Facets of Culture in the Age of Social Transition Proceedings of the All-Russian Research Conference

Date and place: 23-24 March 2018, Ural Federal University named after the first President of Russia B.N. Yeltsin, Yekaterinburg, Russian Federation 
Chief Academic Editor Name:

Signature: fromiri

\section{Knowledge E Publication Ethics and Publication Malpractice Statement}

Knowledge $E$ makes every effort to ensure high quality scholarship in all published articles and conference papers on the KnE Publishing Platform. The platform exists in order to share best practices and accelerate the advance of academic research from regions all over the world. In order to achieve this goal, we need to instill confidence in our readers.

\section{Peer Review}

Each conference whose content is published on the KnE Publishing Platform has undergone academic peer review prior to submission. Knowledge $\mathrm{E}$ checks each submitted paper against a set of criteria to ensure adherence to aims \& scope, prevent ethical or legal concerns before agreeing to publish.

\section{Plagiarism}

Knowledge $E$ verifies the originality of every submitted conference paper submitted using Similarity Check https://www.crossref.org/services/similarity-check/ (powered by iThenticate) to check submissions against previous publications. There is no strict limit or proportion of plagiarized content acceptable however, in cases where manuscripts are submitted for publishing on the KnE Publishing Platform in which the Similarity Check detects more than $20 \%$ similarity then Knowledge E editorial staff ask the academic editor for an explanation and a suitable solution is found. The solution is usually either a re-submission or removal of the paper.

\section{Committee on Publication Ethics (COPE)}


Knowledge $\mathrm{E}$ adheres the policies of the Committee on Publication Ethics (COPE, https://publicationethics.org/), which is a charity registered in the UK. It is concerned with the integrity of peer-reviewed publications in science, particularly biomedicine.

\section{International Committee of Medical Journal Editors (ICMJE)}

Where relevant, Knowledge $\mathrm{E}$ adheres to the policies of the International Committee of Medical Journal Editors (ICMJE), including those that cover overlapping publications. 